This is the author's final, peer-reviewed manuscript as accepted for publication. The publisher-formatted version may be available through the publisher's web site or your institution's library.

\title{
Preferential interaction parameters in biological systems by Kirkwood-Buff theory and computer simulation
}

Myungshim Kang, Paul E. Smith

\section{How to cite this manuscript}

If you make reference to this version of the manuscript, use the following information:

Kang, M., \& Smith, P.E. (2007). Preferential interaction parameters in biological systems by Kirkwood-Buff theory and computer simulation. Retrieved from http://krex.ksu.edu

\section{Published Version Information}

Citation: Kang, M., \& Smith, P.E. (2007). Preferential interaction parameters in biological systems by Kirkwood-Buff theory and computer simulation. Fluid Phase Equilibria, 256(1-2), 14-19.

Copyright: (C) 2006 Elsevier B.V. All rights reserved.

Digital Object Identifier (DOI): doi:10.1016/j.fluid.2006.11.003

Publisher's Link: http://www.sciencedirect.com/science/article/pii/S0378381206004754

This item was retrieved from the K-State Research Exchange (K-REx), the institutional repository of Kansas State University. K-REx is available at http://krex.ksu.edu 


\title{
Preferential Interaction Parameters in Biological Systems by Kirkwood-Buff Theory and Computer Simulation
}

\author{
Myungshim Kang and Paul E. Smith \\ Corresponding author: Paul E. Smith \\ Department of Chemistry \\ 111 Willard Hall \\ Kansas State University \\ Manhattan, KS 66506-3701 \\ Tel: 785-532-5109 \\ Fax: 785-532-6666 \\ Email: pesmith@ksu.edu
}

22 May 2006

\begin{abstract}
Recent results concerning the formulation and evaluation of preferential interactions in biological systems in terms of Kirkwood-Buff (KB) integrals are presented. In particular, experimental and simulated preferential interactions of a cosolvent with a biomolecule in the presence of water are described. It is argued that the preferential interaction parameter defined in a system open to both cosolvent and solvent corresponds to the situation most relevant to the analysis of computer simulation results of cosolvent interactions with proteins and small peptides. Hence, KB theory provides a path from quantities determined from simulation data to the corresponding thermodynamic data.

Keywords: Lysozyme, molecular dynamics, preferential interactions, urea, Kirkwood-Buff theory.
\end{abstract}




\section{Introduction}

Protein denaturation is an important process which remains poorly understood at the atomic level. In principle, computer simulations provide the atomic level detail required for an improved description of cosolvent interactions with proteins. However, the majority of computer simulations of cosolvent effects on peptides and proteins have been rather qualitative in nature.[1-5] In particular, a direct connection between the simulations and experimental thermodynamic data has been noticeably absent. This is primarily a result of the weak binding of many cosolvents to proteins.[6] This presents a conflict between the traditional binding site models used to interpret the experimental data, $[7,8]$ and the inability to locate binding sites and assign binding constants from the simulation data.

More recently, it has become possible to study cosolvent effects in a quantitative manner by the use of Kirkwood-Buff (KB) theory.[9-13] The use of KB theory is particularly well suited for the analysis of experimental data as it involves no approximations, and for the analysis of simulation data as it only requires the determination of radial distribution functions (rdfs), or coordination numbers, which are easily obtained from simulations. Our previous studies have involved using KB theory to improve the force fields required for computer simulation,[14-19] relating simulation data on cosolvent effects to experimental thermodynamic data,[10,11,20,21] and for the interpretation of thermodynamic data on cosolvent effects on biomolecules.[21-24] Here, we present our latest efforts to use $\mathrm{KB}$ theory for the analysis of experimental and computer simulation data relating to the interaction of cosolvents with proteins. The system chosen for study is Lysozyme in urea solutions as extensive experimental data exists for this system. We show 
how this can be used to provide data which is also available from simulation. A comparison of the simulated and experimental data is then performed for the above system using two different force fields for urea.

\section{Background and Theory}

The notation used here follows the usual definitions for biological systems where the subscripts 1,2 , and 3 refer to the primary solvent (usually water), the biomolecule, and cosolvent, respectively. All equations refer to the limit of an infinitely dilute biomolecule. The same formulation can be applied to systems with finite protein concentrations, but is significantly more complicated. The basic approach is to use $\mathrm{KB}$ theory to interpret experimental data from equilibrium dialysis and cosolvent denaturation experiments. The exact details have been outlined elsewhere.[21,23]

Kirkwood-Buff theory provides relationships between particle number fluctuations and derivatives of the chemical potentials in the grand canonical $(\mu \mathrm{VT})$ ensemble where the volume $(V)$, temperature $(T)$, and chemical potential $(\mu)$ of all species are constant. The primary result used here is that,[25,26]

$$
\frac{R T}{V}\left(\frac{\partial N_{i}}{\partial \mu_{j}}\right)_{T, V, \mu_{k \neq j}}=\left(\frac{\partial \rho_{i}}{\partial \beta \mu_{j}}\right)_{T, \mu_{k \neq j}}=\rho_{i} \rho_{j} G_{i j}+\rho_{i} \delta_{i j}
$$

where $\mathrm{G}_{\mathrm{ij}}$ is the Kirkwood-Buff integral between species $i$ and $j, \rho_{\mathrm{i}}=\mathrm{N}_{\mathrm{i}} / \mathrm{V}$ is the number density (molar concentration) of species $i, \mathrm{R}$ is the Gas Constant, $\beta=1 / \mathrm{RT}$, and $\delta_{\mathrm{ij}}$ is the Kroenecker delta function. The KB integrals are defined in terms of the corresponding rdfs ( $\left.\mathrm{g}_{\mathrm{ij}}\right)$ such that, $[21,26]$ 


$$
G_{i j}=G_{j i}=4 \pi \int_{0}^{\infty}\left[g_{i j}^{\mu V T}(r)-1\right] r^{2} d r \approx 4 \pi \int_{0}^{R_{c}}\left[g_{i j}^{N P T}(r)-1\right] r^{2} d r
$$

An excess coordination number can be defined $\left(\mathrm{N}_{\mathrm{ij}}=\rho_{\mathrm{j}} \mathrm{G}_{\mathrm{ij}} \neq \mathrm{N}_{\mathrm{ji}}\right)$ which characterizes the excess number of $j$ molecules around an $i$ molecule in the open system above that observed within an equivalent volume of the bulk reference solution. KB theory can then be used to provide expressions for thermodynamic properties in other ensembles by using suitable thermodynamic transformations.[26]

The approximation in the above equation is required for evaluating $\mathrm{KB}$ integrals in closed systems. Here, a correlation region exists, defined by a distance $\mathrm{R}_{\mathrm{c}}$, within which the local cosolvent and solvent density around the species of interest differs from the bulk density. Beyond the correlation region all $\mathrm{g}_{\mathrm{ij}}(\mathrm{r}) \approx 1$. The correlation region can extend over many molecular solvation shells and therefore provides a potentially different representation of the cosolvent effect from that assumed in the common binding models, where binding is usually limited to the protein surface.

Equilibrium dialysis experiments provide data on the thermodynamic binding of cosolvents to a biomolecule. This data is usually expressed in terms of the preferential interaction (PI) of the cosolvent with the protein (denoted by $\Gamma_{23}$ ), which measures the change in cosolvent molality $\left(\mathrm{m}_{3}\right)$ on changing the biomolecule molality $\left(\mathrm{m}_{2}\right)$ in a system open to the cosolvent and water, but not the biomolecule. This is also often referred to as the preferential binding parameter. In the infinitely dilute biomolecule limit an exact expression for $\Gamma_{23}$ in terms of $\mathrm{KB}$ integrals can be obtained,[23] 


$$
\Gamma_{23}=\left(\frac{\partial m_{3}}{\partial m_{2}}\right)_{T, \mu_{1}, \mu_{3}}=\rho_{3}\left(G_{23}-G_{21}\right)=N_{23}-\frac{\rho_{3}}{\rho_{1}} N_{21}
$$

The value of $\Gamma_{23}$ is dependent on the cosolvent concentration. If the biomolecule exists as a mixture of different major forms (native and denatured for example), the dialysis experiment provides an average preferential interaction such that,

$$
\Gamma_{23}=f_{D} \Gamma_{D 3}+f_{N} \Gamma_{N 3}
$$

where $\mathrm{f}_{\mathrm{i}}$ is the fraction of state $i$. Hence, the total preferential interaction is simply the sum of the individual preferential interactions.

The effects of cosolvents on biomolecules can also be quantified by cosolvent denaturation studies in closed systems. For a biomolecular equilibrium $\left(\mathrm{K}=\mathrm{f}_{\mathrm{D}} / \mathrm{f}_{\mathrm{N}}\right)$ affected by a cosolvent one can show that,[21]

$$
\left(\frac{\partial \ln K}{\partial \ln a_{3}}\right)_{T, P}=\Delta \Gamma_{23}=\Gamma_{D 3}-\Gamma_{N 3}=\Delta N_{23}-\frac{\rho_{3}}{\rho_{1}} \Delta N_{21}
$$

where $\mathrm{a}_{3}$ is the cosolvent activity (on any scale) and $\Delta \mathrm{N}_{2 \mathrm{i}}=\mathrm{N}_{\mathrm{Di}}-\mathrm{N}_{\mathrm{Ni}}$. Hence, the denaturation process is driven by the difference in preferential interaction of the cosolvent with the two different states of the protein. When $\Delta \Gamma_{23}$ is positive, negative, or zero, the cosolvent can be classified as a denaturant, an osmolyte, or thermodynamically inert, respectively. Most denaturation studies use cosolvent concentration and not activity. As the biomolecule is infinitely dilute, the transformation involves a property of the cosolvent and water solution only and can also be expressed in terms of KB integrals according to,[26]

$$
\left(\frac{\partial \ln a_{3}}{\partial \ln \rho_{3}}\right)_{T, P}=\frac{1}{1+\rho_{3}\left(G_{33}-G_{13}\right)}=a_{33}
$$


For our purposes we will also assume that the cosolvent denaturation thermodynamics fits the empirical m-value model such that the change in standard free energy of unfolding is given by,

$$
\Delta \Delta G=\Delta G^{o}\left(\rho_{3}\right)-\Delta G^{o}(0)=-m \rho_{3}
$$

where $m$ is a constant. The approximation is usually good for urea denaturation. Hence, combining Equations 5-7 provides,

$$
\frac{m \rho_{3}}{R T a_{33}}=\Delta \Gamma_{23}=\Delta N_{23}-\frac{\rho_{3}}{\rho_{1}} \Delta N_{21}
$$

where all the data on the far lhs can be obtained experimentally.

A combination of Equations 4 and 5 (or 8 ) can therefore be used to separate the preferential interaction into components involving the native and denatured states as a function of cosolvent concentration. Xie and Timasheff have also described this procedure.[27] The results for Lysozyme in urea solutions at $\mathrm{pH} 7$ (where the protein remains folded) and $\mathrm{pH} 2$ (where the protein unfolds with a transition mid point of $3.7 \mathrm{M}$ urea) have been determined previously.[21,27] The advantage of this approach is that information on cosolvent binding to the native state is available at high urea concentrations where the protein may actually be predominantly unfolded. This is important as the use of computer simulations to study the denatured state is complicated by our limited understanding of the unfolded state of proteins, and our inability to simulate for times long enough to observe unfolding. In contrast, simulations of the native state in high urea concentrations can be performed relatively easily and provide good statistics on the required preferential interactions. This is the approach taken here. An alternative approach 
is to study cosolvent binding at low denaturant concentrations so as to avoid populating the denatured state.[28]

In addition, the individual values of $\mathrm{N}_{21}$ and $\mathrm{N}_{23}$ can be extracted from the above expressions using the $\mathrm{KB}$ results for the partial molar volume $(\bar{V})$ of the solute at infinite dilution in terms of properties of the reference solution,

$$
{\overline{V_{2}}}^{\infty}=R T \kappa_{T}-\rho_{1} \overline{V_{1}} G_{21}-\rho_{3} \overline{V_{3}} G_{23}=R T \kappa_{T}-N_{21} \overline{V_{1}}-N_{23} \overline{V_{3}}
$$

where $\kappa_{\mathrm{T}}$ is the isothermal compressibility of the solution. However, we will not pursue this decomposition here.

\section{Methods}

The details of the simulations will be published elsewhere. Briefly, Hen Egg White Lysozyme was simulated by classical dynamics at $300 \mathrm{~K}$ and $1 \mathrm{~atm}$ using the Gromacs program and the GROMOS 43a1 force field.[29-31] The system included Lysozyme, 4096 urea molecules, and 18112 water molecules in a cubic box of length $9.5 \mathrm{~nm}$. The water model was SPC/E and two different urea force fields (KBFF and OPLS) were investigated.[15,32,33] Positional constraints were applied to the $\mathrm{C}^{\alpha}$ atoms of the protein to prevent partial unfolding. The simulation corresponding to $\mathrm{pH} 7$ was performed with all residues in their usual protonation state, while the simulation at $\mathrm{pH} 2$ was performed with all carboxylate groups protonated. The total simulation time was 6 ns with the final $5 \mathrm{~ns}$ being used for averaging purposes. The properties of the urea and water mixtures have been studied previously and some of the results are displayed in Table 1.[15] The OPLS and 
KBFF models display significantly different urea aggregation behavior, with a high degree of self aggregation (large positive $\mathrm{G}_{\mathrm{ii}}$ values) observed for the OPLS model. We note that the experimental data on Lysozyme denaturation was obtained at $293 \mathrm{~K}$. This is slightly different to the present simulation temperature. We chose $300 \mathrm{~K}$ as the simulated properties of urea and water mixtures are known at this temperature. It is expected that the small temperature difference will have little effect on the results considering the errors inherent in both the experimental $( \pm 1-3)$ and simulated $( \pm 5-10)$ PI data.

The preferential interaction of urea with the protein was determined from the simulations by calculating the number of urea $\left(\mathrm{n}_{23}\right)$ and water molecules $\left(\mathrm{n}_{21}\right)$ within a distance $\mathrm{R}$ from any atom of the protein. This provides a distance dependent PI according to,

$$
\Gamma_{23}(R)=n_{23}(R)-\frac{n_{3}}{n_{1}} n_{21}(R)
$$

The ratio of $n_{3} / n_{1}=\rho_{3} / \rho_{1}$ is the bulk cosolvent to solvent ratio. In cases where the $n_{23}$ and $\mathrm{n}_{21}$ are large, the bulk cosolvent to solvent ratio should be corrected to account for the cosolvent and water molecules which have moved from the bulk reference solution (beyond $\mathrm{R}_{\mathrm{c}}$ ) to the local solution environment around the protein. Hence, a more correct expression is,

$$
\Gamma_{23}(R)=n_{23}(R)-\frac{n_{3}-n_{23}(R)}{n_{1}-n_{21}(R)} n_{21}(R)
$$

The correction involves only a small change in the corresponding bulk concentration ratio, but this can have a significant effect on the calculated PI as $\mathrm{n}_{21}$ can be large. The above analysis is conceptually equivalent to assuming a virtual dialysis membrane located at a 
distance $R_{c}$ from the protein surface. The local volume then represents an open system in contact with a closed NPT particle bath located beyond the correlation distance. The approximation should be reasonable for systems where the bath volume is far larger than the correlation volume. It cannot be used as $\mathrm{R}$ approaches $\mathrm{L} / 2$, where $\mathrm{L}$ is the simulation box length.

\section{Results}

The preferential interaction (PI) of urea with Lysozyme is displayed in Figure 1 for the KBFF and OPLS urea force fields as a function of distance away from the protein surface. The PI is negative at small distances due to the excluded volume effect and the fact that urea is larger than water. The PI then increases sharply due to the presence of an increased number of urea molecules in the first solvation shell, and a corresponding decreased number of water molecules, over that expected from the bulk solution ratio. The OPLS urea model displayed a large preferential interaction of urea with Lysozyme which was several times that of the KBFF urea model, and had not reached the expected plateau value. The correlation volume as defined by $R_{c}$ was also larger $(>1.5 \mathrm{~nm})$ for the OPLS model than for the KBFF model $(1.0 \mathrm{~nm})$. The difference between using Equations 10 and 11 was also more significant for the OPLS model as expected based on their respective PI values.

The experimental value of $\Gamma_{23}$ for this system is determined to be 16 at $\mathrm{pH} 7$ and 10 at $\mathrm{pH}$ 2. The results for the KBFF and OPLS models at $\mathrm{pH} 7$ were 50 and > 200, respectively. Clearly, both urea models display a significantly larger PI than experiment. In 
our opinion, this is almost certainly due to inaccuracies in the protein force field as our previous studies have shown that the approach adopted for common solute force fields used to construct protein force fields do not typically reproduce the experimental KB integrals for solution mixtures.[15,16,19,34] The KBFF model of urea and water does reproduce the experimental $\mathrm{KB}$ integrals (see Table 1) and probably explains the improvement of the KBFF urea model over the OPLS model. However, without a reparametrized protein force field it appears that quantitative agreement with experiment will be difficult.

The effect of $\mathrm{pH}$ on the simulated PI of urea with Lysozyme is also displayed in Figure 1. The simulated effect was small in comparison with the absolute values of $\Gamma_{23}$, and the fluctuations in the instantaneous PI observed during the simulations. As observed in the pH 7 simulations, the simulated PI values were larger for the OPLS model and both models produced values which did not agree with experiment. The decrease in PI on lowering the $\mathrm{pH}$ was reproduced by both models although the PI values remained positive whereas the experimental value is actually negative. However, the small difference observed for the KBFF model was within the statistical errors associated with the simulated PI values.

The rdfs corresponding to the urea and water distribution from the protein surface are displayed in Figure 2. The rdfs indicated a significant interaction of urea with the surface groups on Lysozyme (between 0.3 and $0.5 \mathrm{~nm}$ ) with a smaller second urea shell at larger distances (between 0.6 and $0.8 \mathrm{~nm}$ ). As expected, the water molecules penetrated closer to the protein surface. The differences between the two urea models included an enhanced water interaction over all distances for the KBFF model. A corresponding increase in the urea distribution over a larger distance range was observed for the OPLS 
model. The large differences in the PI values for both urea models were due to the relatively small differences in the rdfs beyond the initial protein contact distance $(0.5 \mathrm{~nm})$, which were magnified upon integration.

Figure 3 displays the PI of urea with Lysozyme as a function of simulation time. The PI increased from around zero (corresponding to a random initial arrangement of molecules) to fluctuate around a larger positive value of the PI. The time history suggests that between 1-2 ns of simulation were required for the urea distribution to equilibrate and provide a reasonable estimate of the PI. This is in agreement with our earlier simulation studies on simple solution mixtures.[35] Long time fluctuations were apparent in the value of the PI which requires averaging over several ns of simulation time to determine PI values with reasonable precision. Slightly longer equilibration times were required for the OPLS urea model.

Most cosolvent molecules are significantly larger than water molecules and cannot therefore approach as close to the protein surface as the water molecules. Consequently, it is well known that this gives rise to an excluded volume effect which tends to stabilize folded proteins. Osmolytes typically enhance the excluded volume effect, whereas denaturants have to overcome the excluded volume effect by binding to the protein in order to promote denaturation. Recently, Schellman has estimated the excluded volume contribution to $\Gamma_{23}$ for several cosolvents and proteins using some simple approximations involving protein solvent accessible surface areas.[36] Estimates for the excluded volume contribution to $\Gamma_{23}$ can also be obtained from the current simulations. From the data provided by Schellman for urea and Lysozyme, the excluded volume (referred to as the gap 
volume by Schellman) for the native protein is $8000 \mathrm{~cm}^{3} / \mathrm{mol}$, if a protein volume of 10100 $\mathrm{cm}^{3} / \mathrm{mol}$ is assumed.[36] This leads to an excluded volume contribution to the PI of -64 in $8 \mathrm{M}$ urea. The excluded volume contribution can be obtained from the simulations as the value of the first minimum in the distance dependent PI (see Figure 1). This provides contributions of -18 for the KBFF and -11 for the OPLS force fields, corresponding to an excluded volume of 2250 and $1375 \mathrm{~cm}^{3} / \mathrm{mol}$, respectively. Clearly, the simulated excluded volume contributions are smaller than the estimates provided by Schellman. This is probably due to the fact that the simulations include protein flexibility and the non spherical nature of urea into the calculation. The degree of excluded volume was also dependent on the urea model and the subsequent interaction with the protein. A larger excluded volume effect was observed with the KBFF model and was consistent with the smaller PI observed for this model.

\section{Conclusions}

It has been shown that calculations of the preferential interaction of urea with native Lysozyme under denaturing conditions provide a convenient way to study cosolvent effects on proteins, especially for comparison of simulated and experimental data. The simulated PI values require several ns of simulation time to equilibrate, and display large fluctuations on the ns timescale. A large positive PI was observed with two different urea models, both of which indicated significantly more urea affinity for the protein than suggested by experiment. In our opinion, the PI represents a model sensitive property of solution mixtures that requires accurate force fields if one desires quantitative agreement 
with experiment.

The difference between the results obtained for KBFF and OPLS urea models was significant. This is not due to the different water model used here (GROMOS and OPLS were developed to be used with the SPC and TIP3P water models), as we have demonstrated that the KB integrals are relatively insensitive to the water model.[14-16] The increased degree of aggregation observed for the OPLS urea model, over the KBFF model, affects the corresponding preferential interactions observed in the presence of an infinitely dilute solute. In an earlier study of cavity formation in urea solutions it was observed that the degree of urea exclusion from the cavity was directly related to the degree of urea aggregation observed in solution; a larger degree of urea aggregation producing a correspondingly larger degree of urea exclusion.[20] Here, the OPLS model describes a more favorable preferential interaction of urea with Lysozyme than the KBFF model. Therefore, it appears that if the urea and water force field is not correctly balanced, leading to incorrect descriptions of self aggregation, this will result in an excessive negative preferential interactions if the solute-cosolvent interaction is unfavorable, and an excessive positive preferential interactions if the solute-cosolvent interaction is favorable. Finally, it should be noted that the general appearance of the urea and water rdfs around the protein are in qualitative agreement for both urea models, even though the thermodynamics are not.

In our opinion, the only way one can be confident in the agreement between experimental and simulated data on preferential interactions is to use both a cosolvent and protein force field which have been shown to accurately reproduce the $\mathrm{KB}$ integrals observed for solution mixtures. 


\section{Acknowledgements}

Acknowledgement is made to the donors of The Petroleum Research Fund, administered by the ACS, for partial support of this research. 


\section{References}

[1] P.E.Smith and B.M.Pettitt, Effects of salt on the structure and dynamics of the bis(penicillamine) enkephalin zwitterion: a simulation study, J.Am.Chem.Soc. 113 (1991) $6029-6037$.

[2] P.E.Smith, G.E.Marlow, and B.M.Pettitt, Peptides in ionic solutions: a simulation study of a bis(penicillamine) enkephalin in sodium acetate solution, J.Am.Chem.Soc. 115 (1993) 7493-7498.

[3] J.Tirado-Rives, M.Orozco, and W.L.Jorgensen, Molecular dynamics simulations of the unfolding of Barnase in water and 8M aqueous urea., Biochemistry 36 (1997) 7313-7329.

[4] A.Caflisch and M.Karplus, Structural details of urea binding to barnase: a molecular dynamics analysis, Structure Fold.Des. 7 (1999) 477-488.

[5] Q.Zou, B.J.Bennion, V.Daggett, and K.P.Murphy, The molecular mechanism of stabilization of proteins by TMAO and its ability to counteract the effects of urea, J.Am.Chem.Soc. 124 (2002) 1192-1202.

[6] S.N.Timasheff, Control of protein stability and reactions by weakly interacting cosolvents: The simplicity of the complicated, Adv.Prot.Chem. 51 (1998) 355-432.

[7] C.Tanford, Protein denaturation. C. Theoretical models for the mechanism of denaturation., Adv.Prot.Chem 24 (1970) 1-95.

[8] J.A.Schellman, A simple model for solvation in mixed solvents. Applications to the stabilization and destabilization of macromolecular structures, Biophys.Chem. 37 (1990) 121-140.

[9] P.E.Smith, Computer simulation of cosolvent effects on hydrophobic hydration, 
J.Phys.Chem.B 103 (1999) 525-534.

[10] R.Chitra and P.E.Smith, Preferential interactions of cosolvents with hydrophobic solutes, J.Phys.Chem.B 105 (2001) 11513-11522.

[11] M.Aburi and P.E.Smith, A combined simulation and Kirkwood-Buff approach to quantify cosolvent effects on the conformational preferences of peptides in solution, J.Phys.Chem.B 108 (2004) 7382-7388.

[12] S.Shimizu and C.L.Boon, The Kirkwood-Buff theory and the effect of cosolvents on biochemical reactions, J.Chem.Phys. 121 (2004) 9147-9155.

[13] I.L.Shulgin and E.Ruckenstein, A protein molecule in an aqueous mixed solvent: Fluctuation theory outlook, J.Chem.Phys. 123 (2005) 054909.

[14] S.Weerasinghe and P.E.Smith, Kirkwood-Buff derived force field for mixtures of acetone and water, J.Chem.Phys. 118 (2003) 10663-10670.

[15] S.Weerasinghe and P.E.Smith, A Kirkwood-Buff derived force field for mixtures of urea and water, J.Phys.Chem.B 107 (2003) 3891-3898.

[16] S.Weerasinghe and P.E.Smith, A Kirkwood-Buff derived force field for sodium chloride in water, J.Chem.Phys. 119 (2003) 11342-11349.

[17] S.Weerasinghe and P.E.Smith, A Kirkwood-Buff derived force field for the simulation of aqueous guanidinium chloride solutions, J.Chem.Phys. 121 (2004) 2180-2186.

[18] S.Weerasinghe and P.E.Smith, A Kirkwood-Buff derived force field for methanol and aqueous methanol solutions, J.Phys.Chem.B 109 (2005) 15080-15086.

[19] M.Kang and P.E.Smith, A Kirkwood-Buff derived force field for amides, J.Comput.Chem. (2006) 27 (2006) 1477-1485. 
[20] S.Weerasinghe and P.E.Smith, Cavity formation and preferential interactions in urea solutions: Dependence on urea aggregation, J.Chem.Phys. 118 (2003) 5901-5910.

[21] P.E.Smith, Cosolvent interactions with biomolecules: Relating computer simulation data to experimental thermodynamic data, J.Phys.Chem.B 108 (2004) 18716-18724.

[22] P.E.Smith, Local chemical potential equalization model for cosolvent effects on biomolecular equilibria, J.Phys.Chem.B 108 (2004) 16271-16278.

[23] P.E.Smith, Equilibrium dialysis data and the relationships between preferential interaction parameters for biological systems in terms of Kirkwood-Buff integrals, J.Phys.Chem.B 110 (2006) 2862-2868.

[24] P.E.Smith, Chemical potential derivatives and preferential interaction parameters in biological systems from Kirkwood-Buff theory, Biophys.J. 91 (2006) 849-856.

[25] J.G.Kirkwood and F.P.Buff, The statistical mechnical theory of solutions. I, J.Chem.Phys. 19 (1951) 774-777.

[26] A.Ben-Naim, Statistical Thermodynamics for Chemists and Biochemists (Plenum Press, New York, 1992).

[27] S.N.Timasheff and G.Xie, Preferential interactions of urea with lysozyme and their linkage to protein denaturation, Biophys.Chem. 105 (2003) 421-448.

[28] B.M.Baynes and B.L.Trout, Proteins in Mixed Solvents: A Molecular-Level Perspective, J.Phys.Chem.B 107 (2003) 14058-14067.

[29] W.R.P.Scott， P.H.Hunenberger， I.G.Tironi， A.E.Mark， S.R.Billeter， J.Fennen, A.E.Torda, T.Huber, P.Kruger, and W.F.Van Gunsteren, The GROMOS biomolecular simulation program package, J.Phys.Chem.A 103 (1999) 3596-3607. 
[30] H.J.C.Berendsen, D.van der Spoel, and R.van Drunen, GROMACS: A message passing parallel molecular dynamics implementation, Comput.Phys.Commun. 91 (1995) 43-56.

[31] E.Lindahl, E.Hess, and D.van der Spoel, Gromacs 3.0: A package for molecular simulation and trajectory analysis, J.Molec.Mod. 7 (2001) 306-317.

[32] H.J.C.Berendsen, J.R.Grigera, and T.P.Straatsma, The missing term in effective pair potentials, J.Phys.Chem. 91 (1987) 6269-6271.

[33] E.M.Duffy, D.L.Severance, and W.L.Jorgensen, Urea: potential functions, log P, and free energy of hydration, Isr.J.Chem. 33 (1993) 1422-1427.

[34] R.Chitra and P.E.Smith, A comparison of the properties of 2,2,2-trifluoroethanol and 2,2,2-trifluoroethanol/water mixtures using different force fields, J.Chem.Phys. 115 (2001) $5521-5530$.

[35] R.Chitra and P.E.Smith, Properties of 2,2,2-trifluoroethanol and water mixtures, J.Chem.Phys. 114 (2001) 426-435.

[36] J.A.Schellman, Protein stability in mixed solvents: A balance of contact interaction and excluded volume, Biophys.J. 85 (2003) 108-125. 


\section{Figure captions}

Figure 1. The simulated distance dependent PI of $8 \mathrm{M}$ urea with native Lysozyme. As a function of urea force field at $\mathrm{pH} 7$ according to Equations 10 and 11 (top). As a function of urea force field and $\mathrm{pH}$ using Equation 11 (bottom). The experimentally observed PIs are 16 at $\mathrm{pH} 7$ and -10 at $\mathrm{pH} 2$.

Figure 2. The urea (top) and water (bottom) rdfs as a function of the closest distance (r) to any protein atom for the two different urea force fields. The simulated data correspond to $8 \mathrm{M}$ urea and a protein at $\mathrm{pH} 7$.

Figure 3. The time history of the $8 \mathrm{M}$ urea PI corresponding to the KBFF model of urea and a protein at $\mathrm{pH}$ 7. The value of $\Gamma_{23}$ was determined for $\mathrm{R}=\mathrm{R}_{\mathrm{c}}=1.0 \mathrm{~nm}$ using Equation 11 . 
Table 1

Simulated properties of $8 \mathrm{M}$ urea in water.

\begin{tabular}{lccccc}
\hline & $\begin{array}{c}\rho \\
\mathrm{g} / \mathrm{cm}^{3}\end{array}$ & $\begin{array}{c}\mathrm{G}_{33} \\
\mathrm{~cm}^{3} / \mathrm{mol}\end{array}$ & $\begin{array}{c}\mathrm{G}_{13} \\
\mathrm{~cm}^{3} / \mathrm{mol}\end{array}$ & $\begin{array}{c}\mathrm{G}_{11} \\
\mathrm{~cm}^{3} / \mathrm{mol}\end{array}$ & $\mathrm{a}_{33}$ \\
\hline KBFF & 1.121 & -39 & -48 & -1 & 0.93 \\
OPLS & 1.130 & 391 & -282 & 128 & 0.16 \\
Exp & 1.119 & -56 & -39 & -5 & 1.16 \\
\hline
\end{tabular}

Data taken from reference 15 